\title{
Archéopages
}

Archéopages

Archéologie et société

Hors-série 3 | 2012

Nouveaux champs de la recherche archéologique

\section{Un lot de céramique singulier dans une sépulture du Chasséen ancien}

Hélène Vergély, Muriel Gandelin et Nicolas Garnier

\section{OpenEdition}

1 Journals

Édition électronique

URL : https://journals.openedition.org/archeopages/477

DOI : 10.4000/archeopages.477

ISSN : 2269-9872

Éditeur

INRAP - Institut national de recherches archéologiques préventives

Édition imprimée

Date de publication : 1 janvier 2012

Pagination : 95-101

ISSN : $1622-8545$

\section{Référence électronique}

Hélène Vergély, Muriel Gandelin et Nicolas Garnier, « Un lot de céramique singulier dans une sépulture du Chasséen ancien », Archéopages [En ligne], Hors-série 3 | 2012, mis en ligne le 01 janvier 2012, consulté le 23 février 2023. URL : http://journals.openedition.org/archeopages/477 ; DOI : https:// doi.org/10.4000/archeopages.477 
connaîtrons, au Néolithique final (Vérazien), les alignements et les alternances de préhensions simples, on peut s'interroger sur une éventuelle paternité du type qui serait ici mise en évidence.

Les fouilles réalisées ces dix dernières années ont donc considérablement fait évoluer les connaissances relatives aux productions céramiques chasséennes de l'Ouest de l'Hérault. En chronologie relative, ces productions constituent, à l'heure actuelle, le meilleur élément de datation des occupations du Néolithique moyen 2 découvertes dans cette zone. Replacées dans le contexte plus large du Chasséen méridional, ces séries viennent compléter ou affiner les séquences définies par ailleurs et s'insèrent parfaitement dans le schéma global du Chasséen méridional, bien qu'elles ne soient pas exemptes de spécificités locales.

GEORJON C., 2003 : «Chronologie, variabilité et phénomènes de récurrence dans les corpus céramiques chasséens de la basse vallée de Lez (Hérault) ", in GASCO J., Gutherz X., LABRIFfe DE P.-A. (DIR.), Temps et espaces culturels du 6ème au $2^{\text {ème }}$ millénaire en France du Sud, Actes des $4^{\text {emes }}$ Rencontres Méridionales de Préhistoire Récente, Nîmes, 28-29 octobre 200o, Lattes, ADALR, p. 115-134.

JÉDIKIAN G., 2004: « Études préliminaires du mobilier céramique », in Loison et al., p. 311-336.

LoIson G., FABRE V., VILLEMEUR I. (DIR.), 2004 : Habitats préhistoriques en bordure de l'Orb, Le Crès, Rocade nord de Béziers. DFS de fouille, Inrap, 3 vol., $430 \mathrm{p}$.

Loison G., Gandelin M., Haurillon R., Gleize Y., Marsac R., VergéLy H., 2011 : «Dynamiques d'occupations des sols à la Préhistoire récente dans la basse vallée de l'Hérault. Les apport de l'A75, tronçon Pézenas-Béziers », in SÉnÉPART I., PERrIN T., Thirault E., Bonnardin S. (DIR.), Marges, Frontières et transgressions, Actualité de la recherche, Actes des 8e Rencontres Méridionales de Préhistoire Récente à Marseille, 7-8 novembre 2008 , Archives d'Écologie Préhistorique, p. 317-343.

VAQUER J., 1991 : « Aspect du Chasséen en Languedoc occidental : habitat et culture matérielle », in Identité du Chasséen, Actes du colloque international de Nemours, 17-19 mai 1989, p. 27-37.
1 L'étude du macrooutillage du site a été effectuée par Caroline Hamon

2 Erl-15185 $=5525 \pm 42 \mathrm{BP}$

soit 4455-4327 avant notre ère.
Un lot de céramique singulier dans une sépulture du Chasséen ancien

Hélène Vergély, Muriel Gandelin

Inrap, UMR 5608 "Travaux et Recherches Archéologiques sur les Cultures, les Espaces et les Sociétés» Nicolas Garnier

Laboratoire d'analyses physico-chimiques

a sépulture 3036, découverte sur le site du Barreau de la Devèze-Sud, contient une

inhumation primaire réalisée dans une fosse de plan oblong, à vocation strictement funéraire, de 1,60 m de long pour 1,05 m de large, creusée dans un substrat miocène fortement induré [Fig.1]. Ce type d'aménagement n'est pas ordinaire, pour le Chasséen méridional, au sein duquel les sépultures installées dans des structures en réemploi sont beaucoup plus fréquentes.

Le mobilier associé au défunt est, à plus d'un titre, remarquable. Il s'agit d'un dépôt composé de pièces complètes : un grand polissoir et un lot de trois céramiques. Le polissoir à plage est de forme quadrangulaire en grès fin micacé, sa surface active présente une concavité particulièrement régulière et totalement lissée $\mathbf{1}$. Il se trouvait dans la partie sommitale du remplissage, de manière oblique, position témoignant probablement d'un dispositif architectural en matière putrescible. Le mobilier céramique, quant à lui, a été découvert dans le même horizon stratigraphique que l'inhumation. Du point de vue typologique, il s'agit de formes rares : un micro-vase ovoïde à lèvre ourlée extérieurement [Fig.2], disposé au pied du défunt, au contact direct d'une coupe à socle ajourée de quatre fenêtres ; face au défunt, se trouvait également une écuelle carénée peu profonde à carène anguleuse basse, ornée de deux plaquettes en sablier diamétralement opposées [Fig.3].

La datation, réalisée sur les restes osseux, est comprise entre 4.455 et 4327 avant notre ère ${ }^{2}$, ce qui permet une attribution de cet ensemble à la phase ancienne du Chasséen. Dans ce contexte chronologique, la présence d'une écuelle carénée constitue déjà un fait notable puisque cette forme est rare avant 4100 avant notre ère. De plus, le décor qu'elle supporte est sans équivalent régional connu. Les deux autres vases sont également d'un type peu fréquent: de façon générale les coupes à socle et les micro-vases chasséens complets sont peu nombreux et presque exclusivement issus de contextes sépulcraux ou cultuels. Toutefois, si la typologie des pièces découvertes dans la sépulture 3036 est remarquable, l'examen attentif de leur mise en forme tend à montrer que, du point de vue technique, elles n'ont pas fait l'objet d'un surinvestissement de la part des potiers. Même si la taphonomie ne permet pas de postuler sur l'aspect éventuellement poli des surfaces, le modelage apparait assez sommaire, voire grossier surtout pour la coupe à socle et, dans une moindre mesure, l'écuelle. Et l'on est en droit de s'interroger sur l'utilisation d'un mobilier à vocation exclusivement funéraire, probablement à usage unique.

L'association du micro-vase et de la coupe à socle étant exceptionnelle, elle invitait à l'élaboration de multiples hypothèses. Nous avons choisi de 

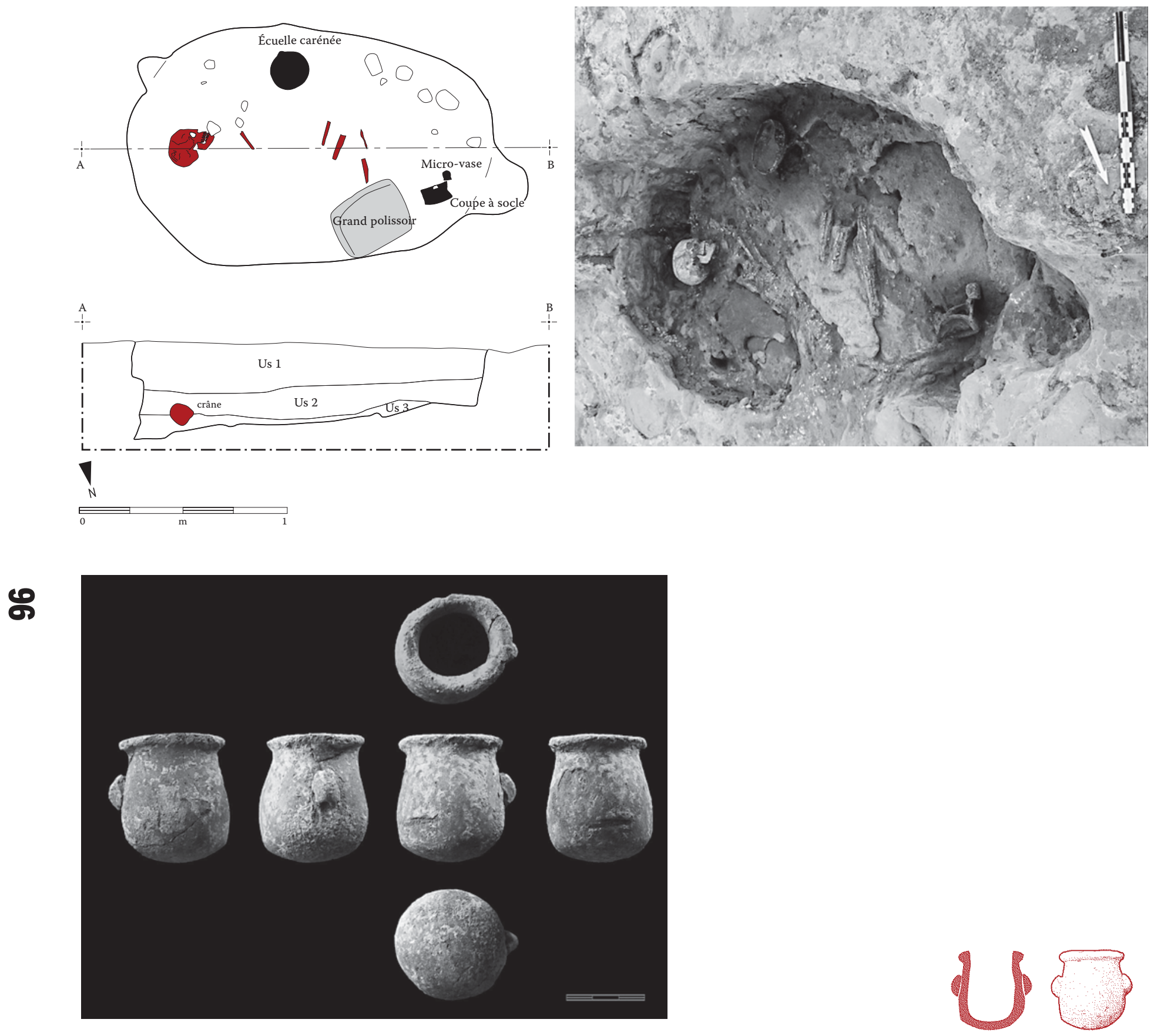

[Fig.1] Relevés et vue de la sépulture 3036, Barreau de la Devèze sud, Béziers (Hérault).

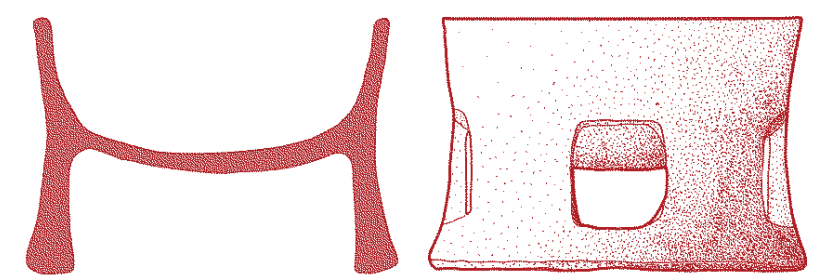

[Fig.2] Micro-vase de la sépulture 3036, Barreau de la Devèze sud, Béziers (Hérault).

[Fig.3] Mobilier céramique d'accompagnement, sépulture 3036, Barreau de la Devèze sud, Béziers (Hérault).

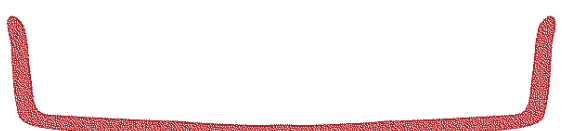

$(2$

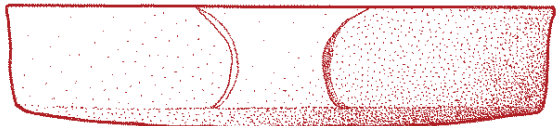


faire appel à d'autres champs de compétences pour nous éclairer, si possible, sur les usages de ces céramiques et les pratiques qui en découlent. Dans cette optique, des analyses chimiques ont été réalisées ${ }^{\mathbf{3}}$. Aucun caramel ou dépôt nétait visible, les résidus de matière amorphe ont été absorbés dans la matrice poreuse de la céramique. La première analyse chimique, réalisée dans le fond interne du micro-vase, a montré qu'il avait contenu une graisse d'origine animale (probablement de ruminant) ; sa transformation chimique indique des chauffages successifs, et un brai ${ }^{4}$ fabriqué à partir de bois de conifère. Une seconde analyse, réalisée sur le réceptacle central de la coupe à socle a montré qu'elle avait contenu différents matériaux organiques. Il s'agit de graisse animale, similaire au micro-vase, et d'une matière grasse d'origine végétale : une huile d'oléastre probablement, ainsi que des traces de résines de conifères ayant subi plusieurs chauffages, et conduisant à la formation d'hydrocarbures poly-aromatiques. Il serait tentant de voir dans l'association de ces deux objets un brûle-parfum et sa réserve. Toutefois, les composés organiques du brai détectés dans le micro-vase et ceux des résines de conifères ne sont pas identiques. Ces résidus ont cependant bien été chauffés, étant donnés les marqueurs de dégradation thermique (hydrocarbures polyaromatiques). Ainsi, si l'étude de ces résidus organiques ne permet pas d'élucider toutes nos interrogations, elle livre des renseignements novateurs sur la gestion des ressources naturelles par les populations du passé, et fournit également, associée à une étude typo-morphologique, des informations inédites sur l'usage, certainement cérémoniel, de céramiques découvertes en contexte funéraire.

\section{Habitat et architecture au Néolithique: l'apport décisif del'archéologie préventive}

Anaïck Samzun

Inrap, UMR 8215 « Trajectoires »

3 Par le Laboratoire

Nicolas Garnier, 63270 Vic-le-Comte. 4. La présence d'acides diterpéniques méthylés et d'hydrocarbures poly-aromatiques écarte l'hypothèse de résine brute, en faveur d'un brai: goudron végétal obtenu par pyrogénation de bois. contribué à renouveler les connaissances et les problématiques liées au Néolithique, ainsi qu'à développer des thématiques relatives à l'organisation des villages, à la fonction de certaines architectures et ont permis d'établir des parallèles avec des occupations contemporaines ailleurs en Europe.

\section{Une appréhension nouvelle des sites} néolithiques. L'approche et les méthodologies de l'archéologie préventive sont sensiblement différentes de l'archéologie programmée, dont les travaux se déroulent généralement pendant plusieurs courtes campagnes annuelles, dans des secteurs limités, dans des contextes environnementaux sélectionnés, et qui ont prévalu pendant une large partie du $\mathrm{xx}^{\mathrm{e}}$ siècle. En revanche, l'accroissement spectaculaire des interventions en archéologie préventive, diagnostics et fouilles, qui sont systématiquement mécanisées, réalisées sur de très larges superficies (parfois plusieurs dizaines d'hectares), dans le cadre d'aménagements extrêmement divers, qu'ils aient lieu en contexte de carrières, d'opérations autoroutières, ferroviaires (les lignes de TGV, LGV...), de canaux (le canal Nord), et même dans des zones urbaines (SaintDenis, Évreux) induisent un changement radical d'échelle pour l'appréhension des sites archéologiques. Ces recherches sont donc entreprises dans tous types de contextes géographiques et géomorphologiques, vallées, versants, plaines et terrasses alluviales, plateaux, zones humides : elles ne privilégient pas un secteur a priori propice, riche en vestiges ou révélé par la carte archéologique, puisque les fouilles préventives liées aux aménagements peuvent finalement concerner tous les terrains et tous les types de zones géographiques. Grâce aux décapages extensifs, elles ont ainsi permis de mettre au jour non seulement des habitats denses et riches en mobilier correspondant à des hameaux ou des villages parfois intégralement préservés et dégagés, des enceintes délimitant des espaces bâtis, mais également de très nombreuses occupations plus modestes (structures éparses, fosses, amas de débitage et aires d'activités diverses...) qui n'en sont pas moins dignes d'intérêt, en particulier quand elles sont attribuables à des étapes peu documentées du Néolithique.

Certaines étapes du Néolithique étaient en effet, jusqu'aux années 2000, très peu étudiées en raison de l'absence même de sites d'habitat, ou reconnues seulement dans des contextes funéraires et ce, sur de larges portions du territoire français. Le Néolithique ancien, qu'il s'agisse du Rubané (vers 5 200-5 000 av. notre ère), puis surtout de la culture de Blicquy-Villeneuve-Saint-Germain (B/VSG), entre 4,900 et 4700 av. notre ère, est en effet assez bien documenté avec, en particulier, l'apparition des toutes premières architectures en terre et en bois reconnues sur notre territoire, qui sont assez aisément décelables lors des opérations de diagnostic et ont été révélées sur de nombreux sites de la moitié nord de la France. En revanche, la situation est très différente pour les étapes moyenne, récente et finale du Néolithique, des étapes du Néolithique sur l'ensemble du territoire français. J. Dubouloz rappelle à son tour que la moitié nord de la France a été pendant longtemps considérée comme un no man's land documentaire pour toute cette période (Dubouloz, 2008). Notre objectif n'est pas ici de proposer un bilan exhaustif des découvertes d'habitats ou d'architectures néolithiques réalisées ces toutes dernières années en archéologie préventive, mais de rendre compte, au travers de quelques études de cas, d'avancées spectaculaires dans la détection, les approches méthodologiques, les études et les interprétations des vestiges mis au jour. Ces progrès ont largement 